Supporting Information:

\title{
Self-Healable Sensors Based Nanoparticles for Detecting Physiological Markers via Skin and Breath: Toward Disease Prevention via Wearable Devices
}

\author{
Han Jin, ${ }^{1,2}$ Tan-Phat Huynh ${ }^{1}$ and Hossam Haick ${ }^{1,3, *}$ \\ 1 The Department of Chemical Engineering, Technion - Israel Institute of Technology, Haifa 3200003, \\ Israel. \\ 2 The Faculty of Information Science and Engineering, Ningbo University, Ningbo 315211, P. R. \\ China. \\ 3 The Russell Berrie Nanotechnology Institute, Technion - Israel Institute of Technology, Haifa \\ 3200003, Israel. \\ * Correspondence to: hhossam@technion.ac.il (H.Haick)
}

\section{Experimental Details}

\subsection{Synthesis of the self-healing polymer substrate: ${ }^{1}$}

4-Aminophenyl disulfide $(750 \mathrm{mg}, 3.0 \mathrm{mmol}$ ) was dissolved in $1 \mathrm{~mL}$ tetrahydrofuran (THF) under ultrasonic conditions. Poly(propylene glycol), tolylene 2,4-diisocyanate terminated, $M_{w}=2300$ (5.1 g, $2.2 \mathrm{mmol}$ ) was added and stirred constantly for $15 \mathrm{~min}$. The resulting viscous mixture was transferred to a Teflon mold and completely degassed under vacuum for $15 \mathrm{~min}$. Finally, the liquid was cured for $48 \mathrm{~h}$ at $75^{\circ} \mathrm{C}$ under vacuum, yielding a product that was a yellowish transparent elastomer. Details of the healing mechanism for the polymer can be found in previously publications. ${ }^{1}$ Briefly, reversible hydrogen (dash line in left side of Figure S1) and covalent (dash line in right side of Figure S1) were formed inside the polymer. After the polymer was scratched, hydrogen and/or covalent bonds were broken. Due to the reversible of hydrogen and covalent bond, the broken bond will automatically recovered and result in the damaged polymer self-healed to its initial state.

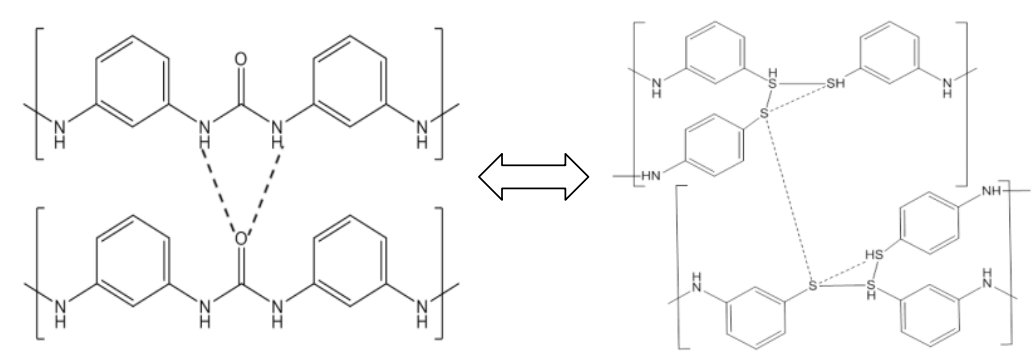

Figure S1. Proposed structural formula of polymer substrate containing reversible hydrogen and covalent bonds. 


\subsection{Preparation of the sh- $\mu \mathrm{Ag}-\mathrm{PU}$ electrode}

$1 \mathrm{~g}$ silver $\mu \mathrm{Ag}(5-8 \mu \mathrm{m}, \geq 99.9 \%$ trace metals basis) powder was dispersed in $220 \mu \mathrm{L}$ polyurethane diol solution $(\mathrm{Mw}=320)$ for $15 \mathrm{~min}$ using a homogenizer. The dark grey viscous $\mu \mathrm{Ag}$-PU composite was degassed under vacuum for $15 \mathrm{~min}$, yielding a highly conductive $(\sim 20$ to $30 \Omega)$ paste.

\subsection{Synthesis of the molecularly functionalized gold nanoparticles (GNPs)}

Molecularly-functionalized 3-5 $\mathrm{nm}$ (in diameter) gold nanoparticles were prepared by a modified two-phase method. ${ }^{2}$ 3-ethoxythiophenol, benzylmercaptan, tert-dodecanethiol, hexanethiol and decanethiol were purchased from Sigma-Aldrich and used as organic ligands. $\mathrm{AuCl}_{4}^{-}$was transferred from an aqueous $\mathrm{HAuCl}_{4} \cdot \mathrm{xH}_{2} \mathrm{O}$ solution $(25 \mathrm{ml}, 31.5 \mathrm{mmol} / \mathrm{L})$; Sigma-Aldrich) to a toluene solution by $80 \mathrm{ml} 34.3 \mathrm{mmol} / \mathrm{L}$ phase-transfer reagent, tetraoctylammonium bromide (Sigma-Aldrich). After the organic phase had been isolated, an excess of organic ligands were added to the solution. The molar ratio of thiol : $\mathrm{HAuCl}_{4} \cdot \mathrm{xH}_{2} \mathrm{O}$ was varied from $1: 1$ to $10: 1$ depending on the kind of thiol in order to prepare a monodispersed solution of gold nanoparticles of average size (3-5 nm). After vigorous stirring of the solution for $10 \mathrm{~min}$, an aqueous solution of reducing agent, $\mathrm{NaBH}_{4}$ (Sigma-Aldrich), in a large excess ( $25 \mathrm{ml}, 0.4 \mathrm{~mol} / \mathrm{L}$, ice-cooled) was added. The mixture was stirred at room temperature for $>3 \mathrm{~h}$, producing a dark brown solution of the thiol-capped gold nanoparticles. The solvent was removed in a rotary evaporator, followed by several washes in ethanol and toluene.

\subsection{Fabrication of the self-healable and flexible nanoparticle-based sensor array ${ }^{1}$}

For fabrication of a self-healable nanoparticle-based flexible sensor array, a $100 \times 100 \times 1$ $\mathrm{mm}$ polymer sheet was prepared as a substrate. A $50 \mu \mathrm{m}$ non-adhesive paper mask placed onto this substrate was coated with sh- $\mu \mathrm{Ag}-\mathrm{PU}$ electrodes using a screen-printing method. ${ }^{1}$ After removing the mask, the distance between two sh- $\mu \mathrm{Ag}$-PU electrodes was $0.5 \mathrm{~mm}$. Two electrical wires penetrating through the back of the sh-crl-PU substrate were in connection with the sh- $\mu \mathrm{Ag}-\mathrm{PU}$ electrodes. This structure was evacuated for $30 \mathrm{~min}$ before drop-casting $1 \mu \mathrm{L} 50 \mathrm{mg} / \mathrm{mL}$ solution of different molecularly-functionalized GNPs onto the groove between the two sh- $\mu \mathrm{Ag}$-PU electrodes. Hence, molecularly-functionalized GNPs were firmly stuck to the substrate, due to the sticky surface of polymer substrate. Finally, the sensor array was stored under vacuum for $1 \mathrm{~d}$ before measurement.

\subsection{Evaluating the sensor array in monitoring of pressure variation}

A MARK 10 ESM301 motorized test-stand was used to apply a strain at a constant speed of $0.05 \mathrm{mms}^{-1}$. The stress was applied by an upper beam (downward) and supported by bottom beams (stationary), under which the substrate bent. The outer (upper) surface became compressed, while the inner (lower) surface was dilated. In the stretching set-up, the 
strain/force was applied between two metal grips on a dog bone sample. The strain was applied as above between metal grips attached to the wider part of the sample, whereas most of the strain was taken by the thinner part of the sample. The forces were measured by an advanced digital force gauge (Mark10, USA). Electrical resistance in these experiments was measured by a Keithley data-logger device (model 2701 DMM) controlled by a custom Labview program. The conversion of gram-force ( $\mathrm{gf}$ ) to $\mathrm{gf} / \mathrm{cm}^{2}$ is: $\mathrm{gf} / \mathrm{S}$, where the $\mathrm{S}$ is the area of the sensor, in this research the $S$ is $2 \mathrm{~cm}^{2}$ (length $\times$ width: $20 \times 10 \mathrm{~mm}$ ). As for the conversion of $\mathrm{gf} / \mathrm{cm}^{2}$ to $\mathrm{mmHg}$, it can be performed through website: http://www.endmemo.com/sconvert/gf cm2mmhg.php

\subsection{Evaluation of the sensor array toward 11 kinds of VOCs}

A Keithley data-logger device (model 2701 DMM) controlled by a custom Labview program was used to sequentially acquire resistance readings from the sensor array and voltage readings from the environmental sensors. A typical exposure cycle involved a 5 min vacuum ( $<100$ mTorr) baseline step, followed by $5 \mathrm{~s}$ exposure and $4 \mathrm{~min} 55 \mathrm{~s}$ measurement of the test vapor under still conditions, ending with another 5 min vacuum step. Nitrogen and $11 \mathrm{kinds}$ of VOC flow (5 L/min constant flow rate) were alternately introduced into the chamber (Table S1). Four successively increasing concentrations of each VOC, controlled gas concentrations (0.5-20 ppm), were produced by a commercial dynamic liquid injection dilution system (Umwelttechnik MCZ, Germany). ${ }^{3}$ The power of the sensor array could be calculated from the equation $U^{2} / R$, where the $U$ is the applied voltage on the sensor array ( $5 \mathrm{~V}$ in this case), and $R$ is the resistance of the sensor array after exposure to the target gas.

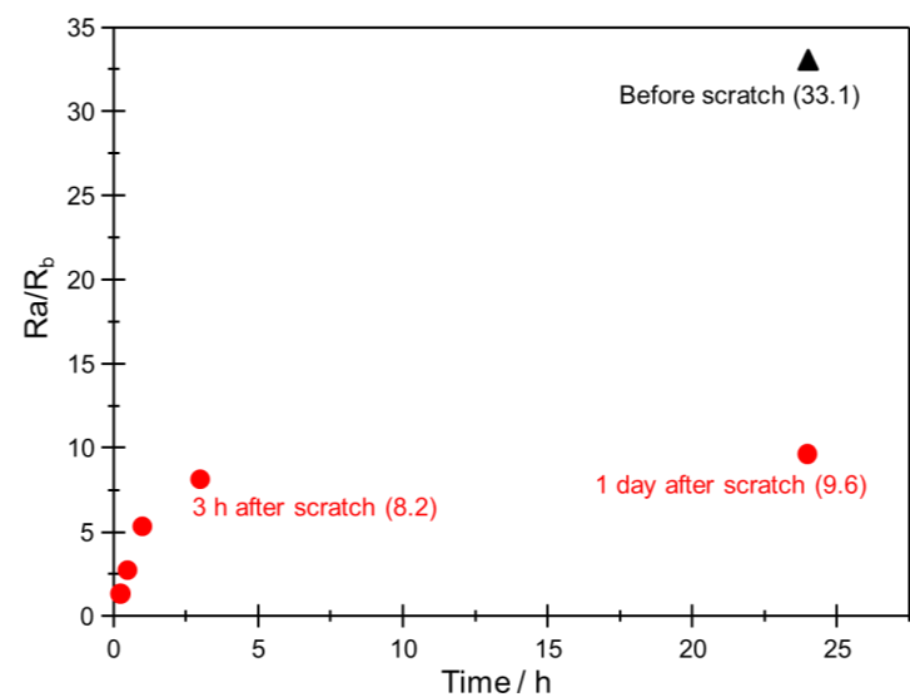

Figure S2. Response of the sensor unit consisting of benzylmercaptan-functionalized GNP film to 20 ppm hexanal in a scratching/healing cycle. 
Table S1 Healing period and sensing response (to $20 \mathrm{ppm}$ hexanal) of the molecularly-functionalized GNP at different concentration.

\begin{tabular}{|c|c|c|c|c|c|c|c|c|c|c|c|c|c|c|c|}
\hline \multirow{3}{*}{$\begin{array}{l}\text { Ligands-functionalized } \\
\text { GNP } \\
\text { Concentrations / (mg/mL) }\end{array}$} & \multirow{2}{*}{\multicolumn{3}{|c|}{$\begin{array}{l}\text { 3-Ethoxythiophenol } \\
\text { functionalized GNP }\end{array}$}} & \multirow{2}{*}{\multicolumn{3}{|c|}{$\begin{array}{l}\text { Benzylmercaptan } \\
\text { functionalized GNP }\end{array}$}} & \multicolumn{3}{|c|}{ Tert-dodecanethiol } & \multicolumn{3}{|c|}{ Hexanethiol } & \multirow{2}{*}{\multicolumn{3}{|c|}{$\begin{array}{c}\text { Decanethiol } \\
\text { functionalized GNP }\end{array}$}} \\
\hline & & & & & & & & nalized & GNP & functi & nalized & GNP & & & \\
\hline & 10 & 20 & 50 & 10 & 20 & 50 & 10 & 20 & 50 & 10 & 20 & 50 & 10 & 20 & 50 \\
\hline Healing period / $\mathrm{h}$ & 3 & 3 & 3 & 3 & 3 & 3 & 3 & 3 & 3 & 3 & 3 & 3 & 3 & 3 & 3 \\
\hline $\begin{array}{l}\text { Response }\left(\Delta R_{a} / R_{b}\right) \text { to } \\
20 \mathrm{ppm} \text { Hexanal } / \%\end{array}$ & 1.60 & 2.21 & 3.05 & 6.01 & 15.04 & 36.77 & 2.04 & 4.05 & 10.94 & -4.49 & -9.75 & -15.63 & 1.36 & 3.11 & 6.78 \\
\hline
\end{tabular}

The healing capability of the sensing films in different concentration is analogous, but their sensing performance is decreased with the decreasing of concentration of the sensing films. The declining of the performance for the sensing films at low concentration can be attributed to the inadequate sensing chemicals for sensing of VOCs (e.g. hexanal).

Table S2 Chemical formula and physical properties of the selected 11 kinds of VOCs found in human breath/skin.

\begin{tabular}{|c|c|c|c|c|c|c|}
\hline VOCs & CAS NO. & Formula & Boiling point $\left({ }^{\circ} \mathrm{C}\right)$ & Density $\left(\right.$ at $\left.25^{\circ} \mathrm{C}\right)\left(\mathrm{g} / \mathrm{cm}^{3}\right)$ & $\mathrm{P}_{0}\left(\right.$ at $\left.25^{\circ} \mathrm{C}\right)(\mathrm{kPa})$ & Breath/Skin \\
\hline 2-Hexanone & $591-78-6$ & & 158.1 & 0.830 & 1.5 & Breath/Skin \\
\hline Acetic acid & $64-19-7$ & & 117.9 & 1.0446 & 2.1 & Breath/Skin \\
\hline Heptane & $142-82-5$ & & 98.4 & 0.6795 & 6.0 & Breath/Skin \\
\hline Hexanal & $66-25-1$ & & 160.5 & 0.8335 & 1.4 & Breath/Skin \\
\hline n-Hexane & $110-54-3$ & & 68.7 & 0.6606 & 20.4 & Breath/Skin \\
\hline 3-Heptanone & $106-35-4$ & & 170.3 & 0.8183 & 0.5 & Breath \\
\hline Heptanotic acid & $111-14-8$ & & 249.2 & 0.9181 & $1.9 \times 10^{-3}$ & Breath/Skin \\
\hline Hexanoic acid & $142-62-1$ & & 205.8 & 0.929 & $5.7 \times 10^{-3}$ & Breath/Skin \\
\hline Heptanal & $111-71-7$ & & 152.8 & 0.8216 & 0.5 & Breath/Skin \\
\hline Nonanal & $124-19-6$ & & 209.7 & 0.8264 & $3.6 \times 10^{-2}$ & Breath/Skin \\
\hline Nonane & $111-84-2$ & & 151 & 0.7173 & 0.5 & Breath/Skin \\
\hline
\end{tabular}


Before scratch: intimate contacts between GNP in the film

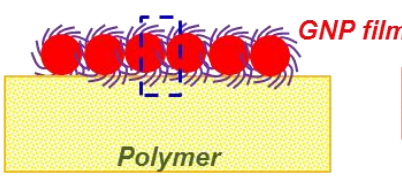

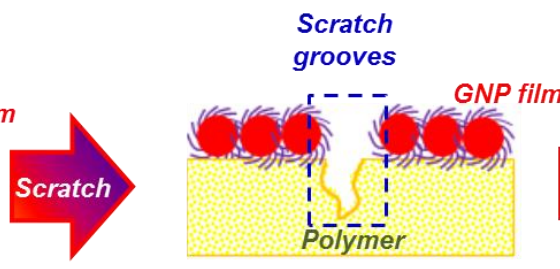

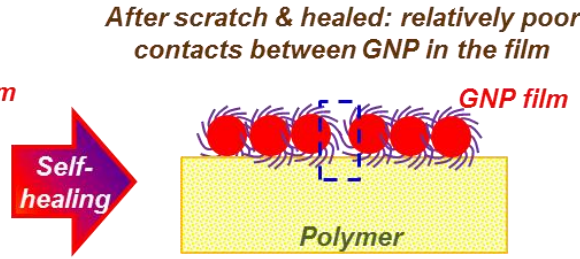

Figure S3. Schema of the contacts for GNP (in the film) before and after scratch. Subsequently, the contacts between GNP in the film became relatively poor, leading to the performance of the sensor being reduced to $<100 \%$ after full healing.
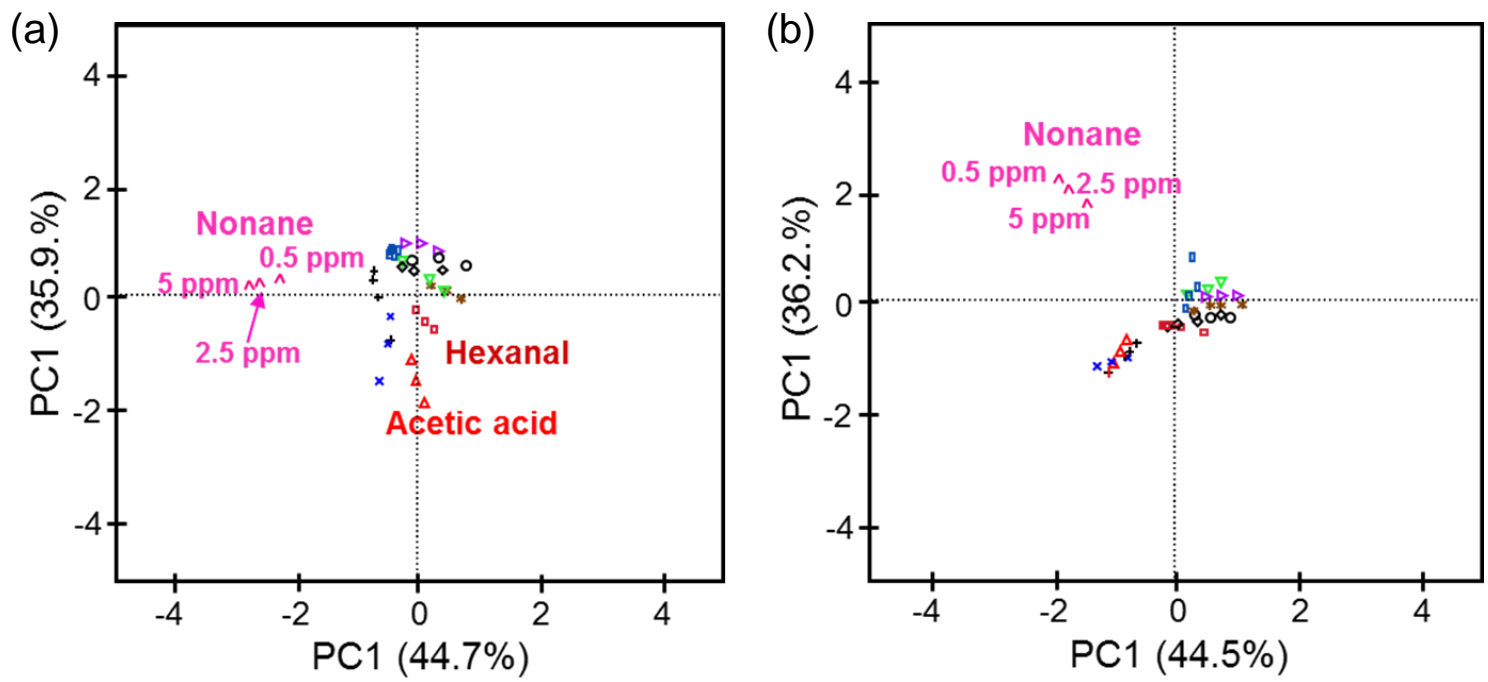

Figure S4. PCA schemes of the dataset for the sensor array in sensing of VOC in the range of 0.5-2.5 ppm: (a) after the second and (b) the third cycle of scratching/healing. After the second and third cycle of scratching/healing, the sensor array lost $73 \%$ of its original performance (3 VOCs can be discriminated) and $90 \%$ (only nonane could be discriminated) discrimination feature to the examined VOCs.

\section{References:}

1. Huynh, T. P.; Haick, H. Adv. Mater. 2016, 28, 138-143.

2. Brust, M. F., J.; Bethell, D.; Schiffrin, D. J.; Kiely, C. J. J. Chem. Soc. Chem. Commun. 1995, (16), 1655-1656.

3. Bayn, A.; Nol, P.; Tisch, U.; Rhyan, J.; Ellis, C. K.; Haick, H. Anal. Chem. 2013, 85, 11146-11152. 